\title{
Cataract Image Analysis System
}

\begin{abstract}
A system for sequential colour video capture and analysis of lens opacities is described. A sensitive RGB (Red-Green-Blue) camera is coupled to a 486 DX2/66 IBM-compatible computer to obtain high-resolution images of cataracts. Vacuoles, retro-dots, suture opacities, wedge opacities, cortical and posterior subcapsular opacities and nuclear sclerosis can be digitised and subjected to various analysis systems and colour prints obtained. The size and number of vacuoles and opacities can be determined by selecting areas of interest, and line tracings of whole lens, isolated opacities or nuclear sclerosis can be separated by analysis into 16.8 million plus colour gradients. The Cataract Image Analysis System represents a precise and new digitised procedure for cataract documentation and may be of value in determining cataract progression and evaluation of medical therapy.
\end{abstract}

Key words Ageing, Cataract, Crystalline lens, Image analysis, Nuclear sclerosis, Refraction

The transparency of the crystalline lens of the eye is essential to preserve clear vision and focusing of light images on the retina. Opacities of the crystalline lens - cataract(s) - result in distorted or poor vision. The most common type of cataract is found in humans after 60 years of age (age-related cataracts). The opacities of the ageing human lens are located in the anterior or posterior cortex, in the nucleus or under the capsular areas. In most human cataracts there are various combinations of opacities. Several methods for either photography and optical or visual estimation of opacities are available: (a) slit lamp photography, (b) retro-illumination photography and densitometry with visible light, (c) Scheimpflug photography and densitometry, (d) optical degradation of projected retinal images, (e) fluorimetry, and (f) laser quasielastic light scattering (all summarised in references 1-6). Black and white images from slit lamp or retro-illumination video pictures of the lens have been subjected to image analysis. ${ }^{7-9}$ The latter systems do not document the colour and area-colour of lens opacities, the areas of water clefts or vacuoles and do not allow for precise densitometric measurements behind the areas of existing opacities. Also three-dimensional (3D) reconstruction of the size and position of the opacities have not been documented. The method described here represents a precise and new quantitative procedure for short- and longterm documentation of opacities in the lens that could be of value for studies on the natural progression of cataracts and evaluation of medical therapy of cataracts.

\section{Material and method}

The method described here is based on the principle that transmitted and reflected light of focal-plane images can be used for 3D imaging and density determinations. In a transparent tissue, such as the crystalline lens, only higherdensity structures will reflect light or obstruct the passage of retro-illuminated light. For each single image plane, reflected light units $(R u)$ correspond to the intensity of illumination (I), the reflectance of the structures $(R S D)$ and the optimum illumination capture angle $\left(I_{\mathrm{o}}\right)$ as follows: $R u=I \times R S D \times I_{\mathrm{o}}$.

Structures that do not reflect light will, in the single image plane, appear dark. Thus, the contrast of reflected versus non-reflected light allows determination of structural density in the reflected areas. The structural density in the reflected areas can thus be analysed to reconstruct the shape, form and contours of such areas. The new procedure, Cataract Image Analysis System (CIAS), consists of recording the colour, shape and location of the human cataract opacities by high-resolution RGB (Red-Green-Blue) video, and applying digital analysis computer-based programming to specific quantification and classification of such opacities and areas of optical discontinuity. Light reflectance by opaque areas of the human lens allows 3D reconstruction of the cataract areas and correlation in high-resolution video images and colour prints. An additional method of optical parallax and/or modification of image brightness/contrast percentages by image processing allows 3D visualisation of opacities in the crystalline lens. Finally, image analysis of similar colour or fluorescent areas in
E. Cotlier

New York State Institute for Basic Research in Developmental Disabilities New York, USA

Edward Cotlier, MD 372 Fifth Avenue

New York NY 10018, USA

Tel: +1 (212) 9477023 Fax: +1 (212) 2790798 


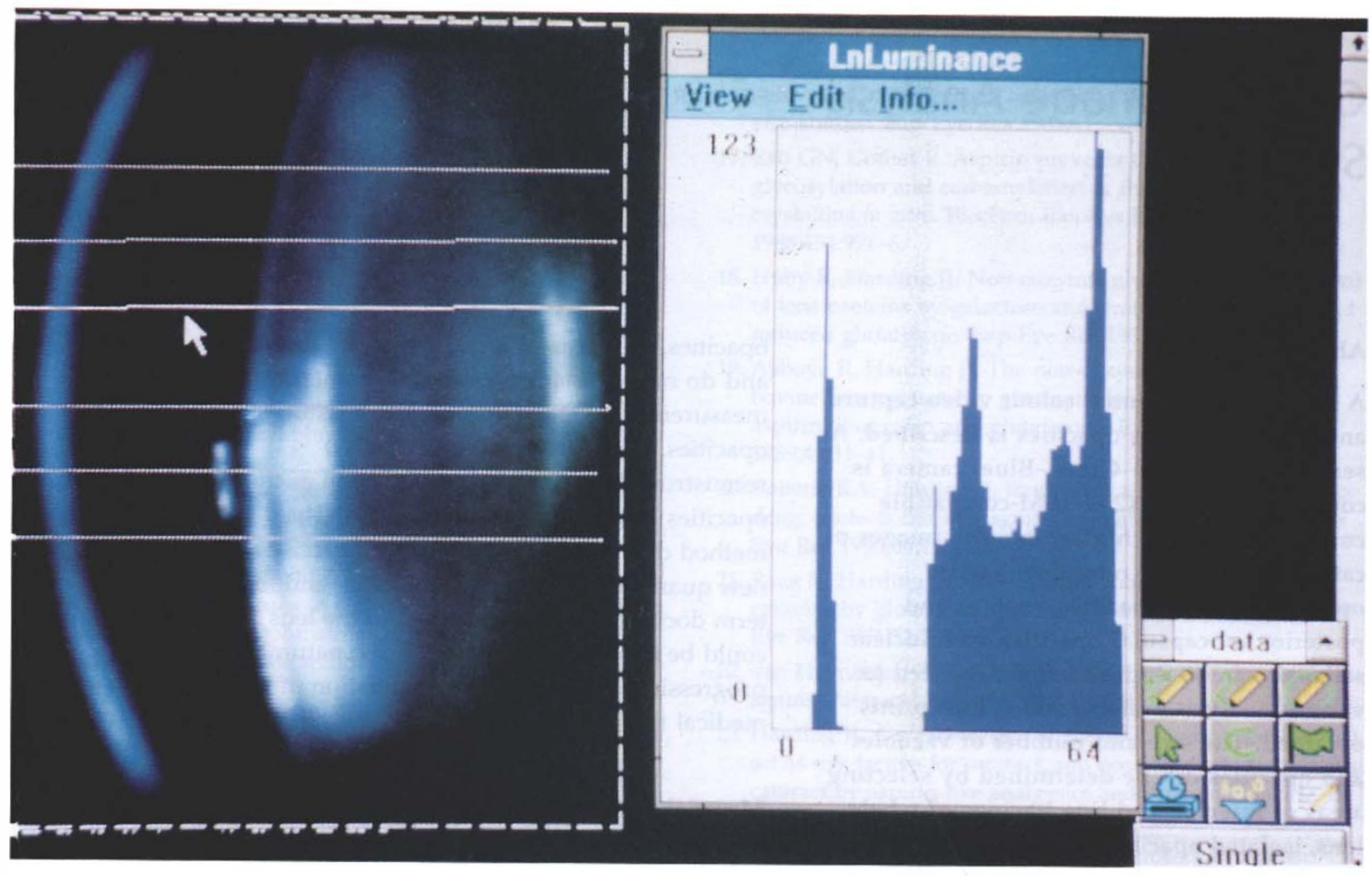

(a)

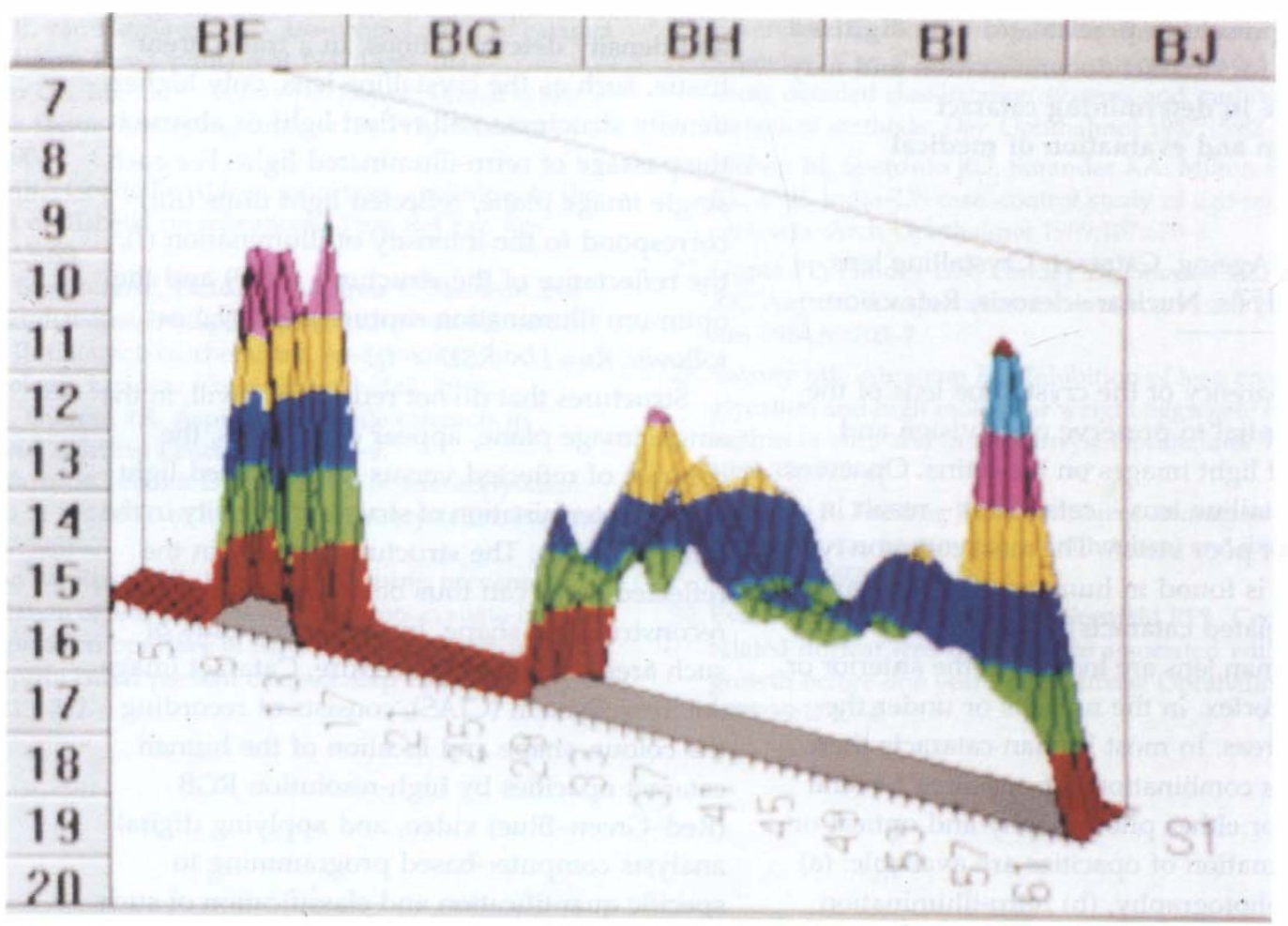

(b)

Fig. 1. (a) Six line tracings through a sagittal section of anterior cortical and posterior subcapsular cataract. The arrow points to the line analysed in the histogram. (b) The Excel-exported six lines with separate colour coding for each line tracing and the corresponding luminance.

the lens nucleus or cortex permits determination of area surface (in square millimetres or square micrometres) and plotting of histograms to determine temporal modifications in cataracts.

\section{Camera, software and analysis system}

Image capture is performed with a high-resolution video analogue RGB remote-head microscope camera attached to a Topcon SL-7 slit lamp. The camera is preset by auto 


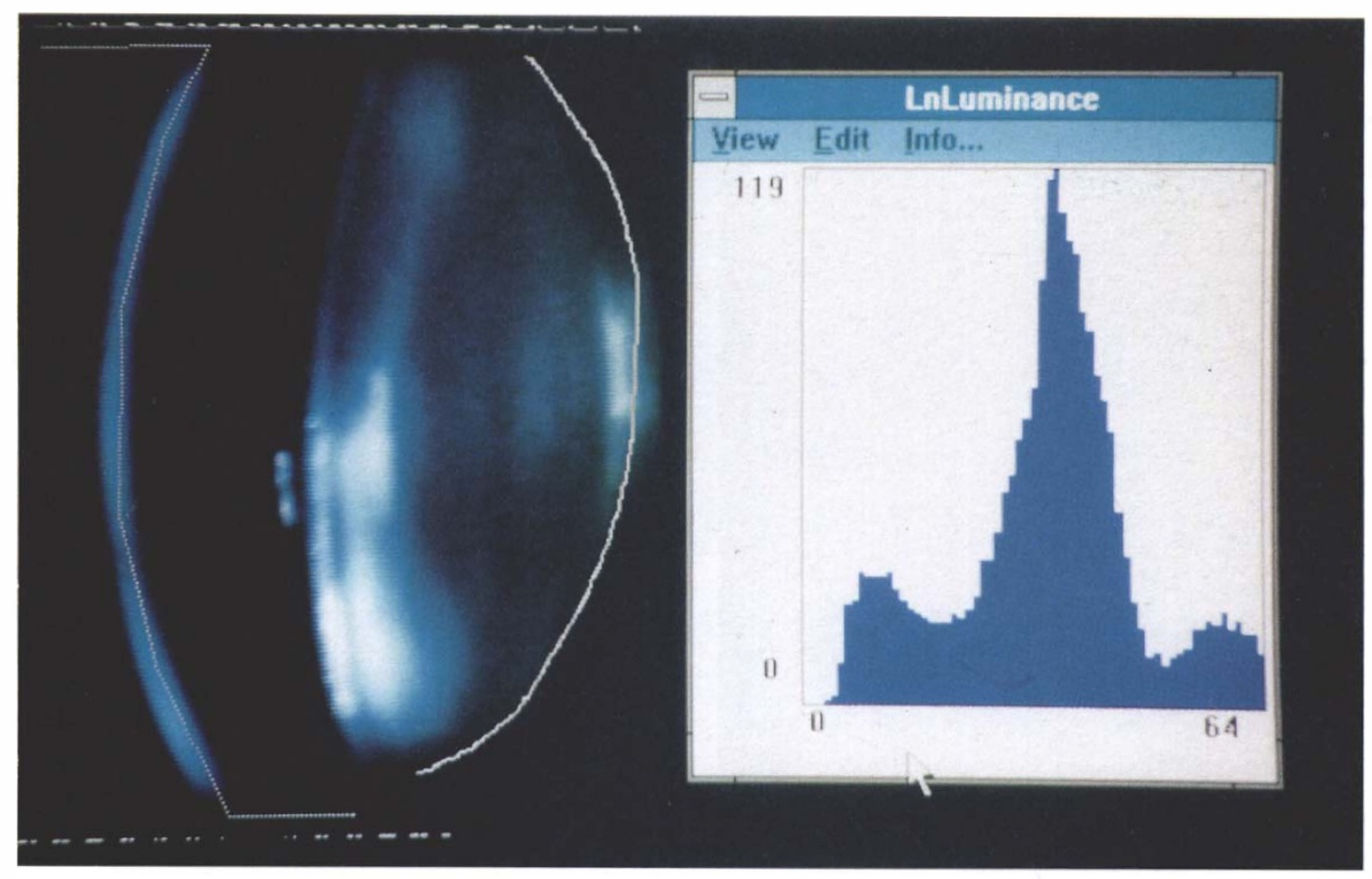

(a)

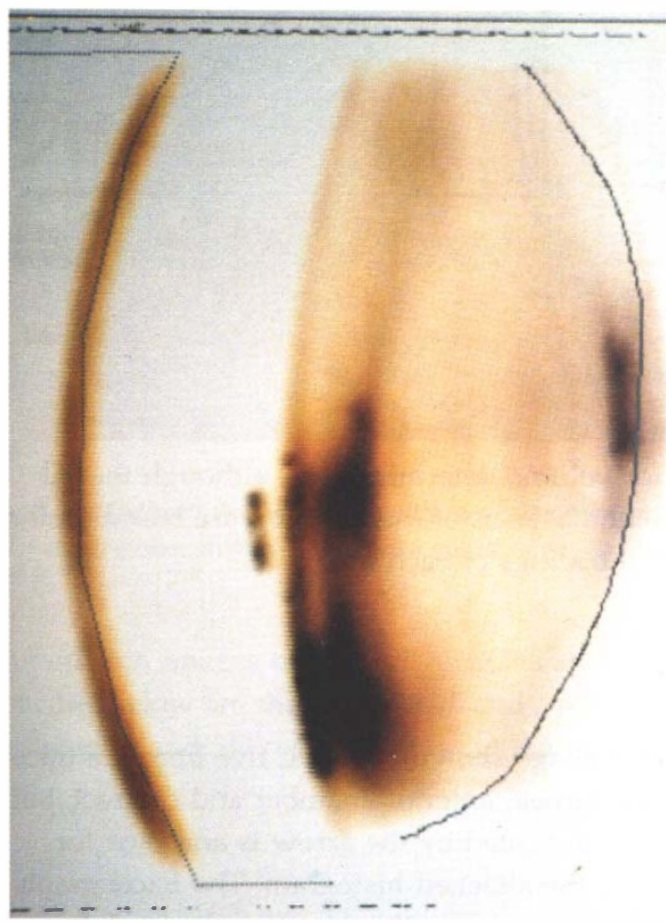

Fig. 2. (a) A circular tracing through the post-subcapsular area of the cataract in Fig. 1 with its corresponding histogram. (b) A negative image of the same eye.

(b)

exposure to allow video observations and recordings in bright and dark fields, polarising, fluorescent and confocal systems. The camera is sensitive to 0.003 lux at fl.2, 30ire output and $+12 \mathrm{~dB}$ gain and elicity NTSC colour or PAL colour. This analogue signal is then sent to an analogue-to-digital converter with frame grabber. This frame grabber is installed in a 486DX2/66 IBMcompatible computer. The frame grabber is controlled by software running under a Windows operating system. Statistics and graphics are performed in Microsoft Excel (Seattle, WA). The images are then printed with the use of a dye sublimination colour video printer. Image analysis is performed with the Image-Pro Plus program (Media Cybernetics, Silver Spring, MD).

Images are captured by rotating the side arm of the slit lamp or the camera from 0 to 90 degrees. The pinhole diaphragm in front of the camera increases the depth of focus in such a way that low-magnification images obtained at greater than 60 degree angles do not require changes in the camera plane as in the Scheimpflug Corrected Cameras (SCC). The slit lamp illumination with halogen bulb is controlled by a preset rheostat and 


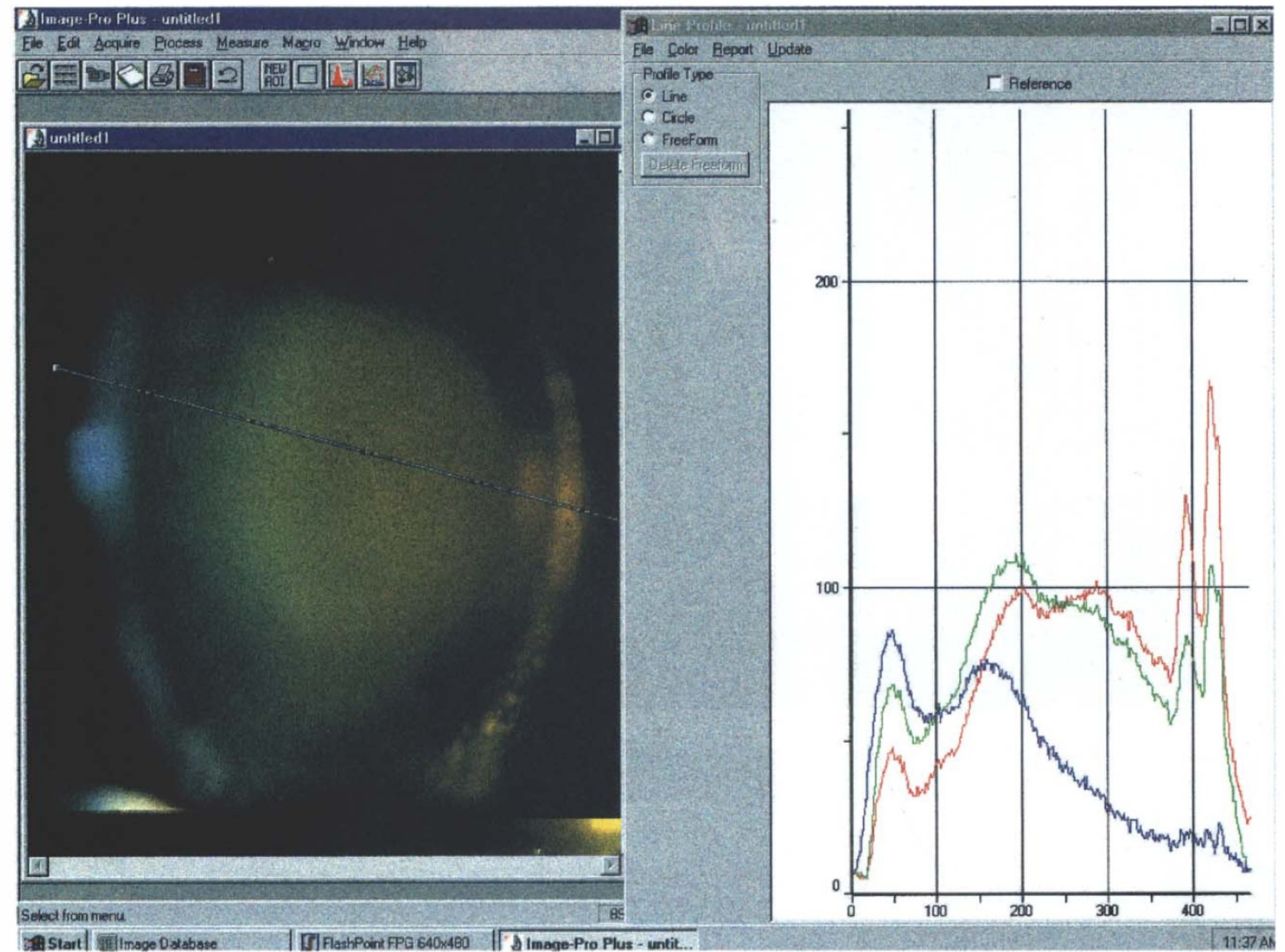

Fig. 3. A line tracing through a mixed cataract: anterior cortical, nuclear sclerosis and double posterior subcapsular opacities (left-hand side) subjected to colour analysis and the isolated red, green and blue components (right-hand side).

slit beam. Values for each setting are recorded for use at precisely the same settings in subsequent image captures.

Three major image captures were performed: (1) Sagittal sections of the lens in the anteroposterior plane were analysed as a whole or divided into an anterior and posterior segment. These sections were particularly suitable for analysis of cortical and posterior subcapsular opacities and nuclear density. The sagittal sections at high magnification provided a view of the cortical sutures and their enlargement during cataract formation. (2) Retro-illumination images obtained at 5 degrees or less. These images were particularly suitable for posterior subcapsular cataracts, vacuoles or retro-dots and linear or cuneiform cortical opacities. (3) Images of opacities obtained by direct or side illumination. These images were used for cortical vacuoles or clefts and for linear or circular cortical ridges (Obazawa circular ridges).

Images were then subjected to (a) either single or multiple line tracings (black and white or colour) and (b) $3 \mathrm{D}$ reconstruction. The Image-Pro Plus program contains a variety of measuring tools that allow the determination of distances within a line or area that has been preselected. Distances can be quantitated in micrometres and areas in square micrometres. It is not, however, suitable for volume determinations, although the 3D reconstruction aspects of the program are based on the geometrical tracings of each image.

\section{Results}

In the video image shown in Fig. 1, five lines are traced through the cornea, anterior chamber and cataract, but only the line indicated by the arrow is analysed for luminance in the attached histogram. The Excel graph shown in Fig. 1b indicates the densities obtained through the various tracings. Fig. 2 shows a curved line through the posterior subcapsular cataract with the corresponding luminance histogram and their reversed image in Fig. 2b. Fig. 3 shows a sagittal section of the lens with the line tracing separated into the red, green and blue components. Note that the double posterior subcapsular opacities shows two peaks for the red and green components of the densitogram but no significant density increase on the blue component. By direct illumination of cortical opacities, the white wedge mountain-like peaks could be documented in the anterior cortex and their yellow counterparts in the posterior cortex (Fig. 4). A line tracing is shown in Fig. 4a that corresponds to the 3D reconstruction in Fig. 4b. Retro- 

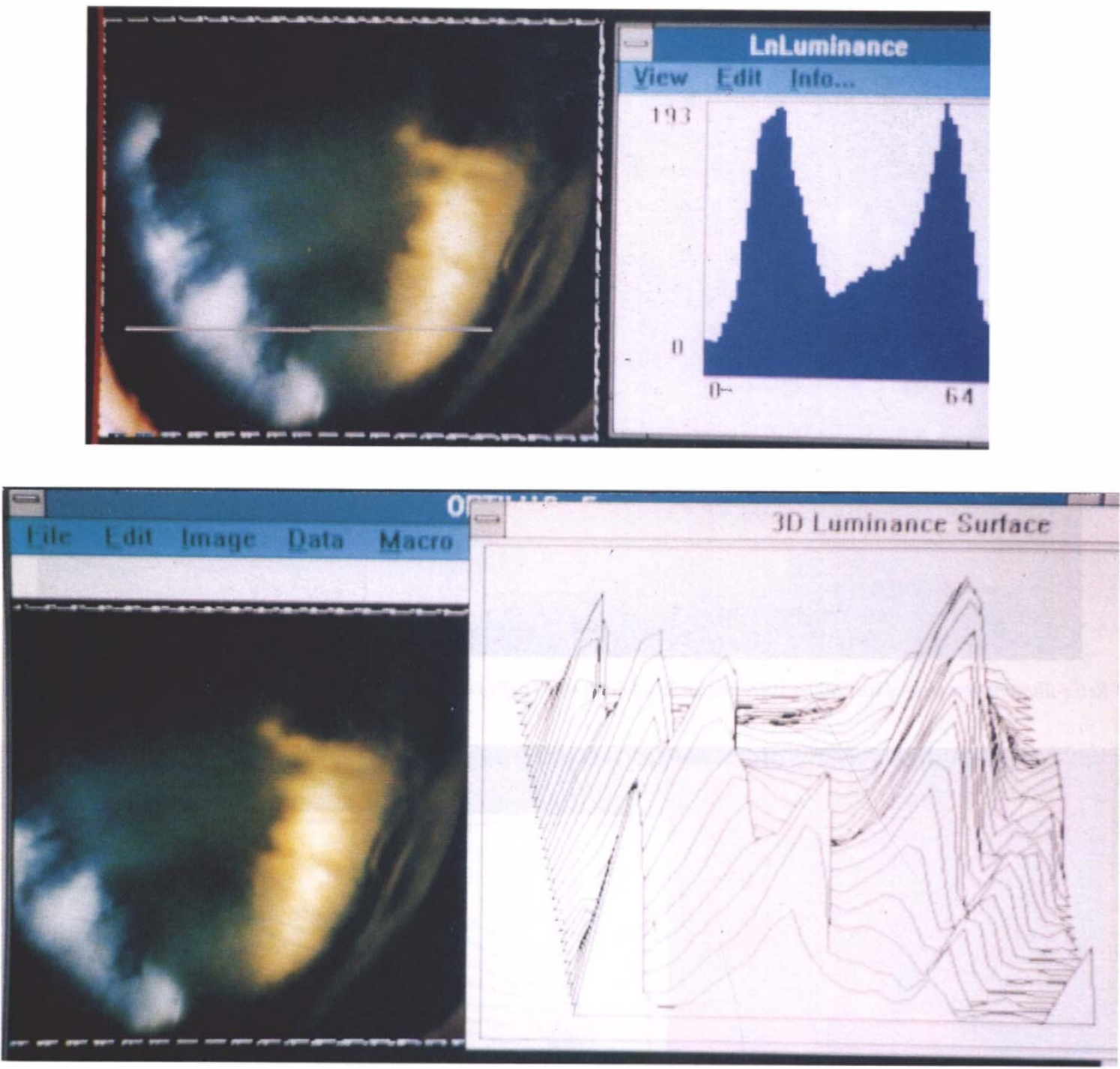

(b)

Fig. 4. (a) Indirect illumination of white wedge anterior cortical opacities and yellow posterior cortical opacities sectioned by line tracing (left-hand side) and the corresponding luminance profile (right-hand side). (b) Three-dimensional reconstruction of $(a)$.

illumination images of cortical vacuoles and spoke opacities below are shown in Fig. 5 and a magnified view of vacuoles and 3D reconstruction in Fig. 6.

\section{Discussion}

Image analysis of cataracts requires digitised images of opacities and vacuoles in the cortex and posterior subcapsular area and colour densities of the nucleus. Cortical and nuclear opacities have previously been obtained by a combination of Scheimpflug sagittal plane photography and retro-illumination. 2,3,6 These data are valuable for repeated measurements, but only the central plane of the lens is analysed at low magnification with the Scheimpflug sagittal plane images. Analysis of nuclear colour densities has been performed by visual estimation of colour photographs. ${ }^{5}$ However, there are problems concerning reproducibility of colour in different film batches or film development procedures.
The use of a digital colour camera coupled to colour image analysis represents a new approach to documentation of lens opacities. Particularly suitable is the quantitation of colour in nuclear sclerosis by analysis of line tracings with the three major colour hues: red, green and blue. Digitised colours are accurately reproduced and can be quantitated in luminance gradients of red, green and blue to 16 million plus combinations. The advantage of a digital colour camera, which is easy to standardise, is the storage of digital data in diskettes for subsequent analysis and the simultaneous review of several sections of the lens. In addition, areas of each opacity, lens vacuoles or water clefts can be estimated.

The quantitation and listing of the number and size of lens opacities (dots, wedges and ridges) requires standardisation and direct access to Excel, through the Image-Pro Plus program used in the CIAS. In subsequent papers, automation set up for image capture and immediate processing through macro programs will be described. 


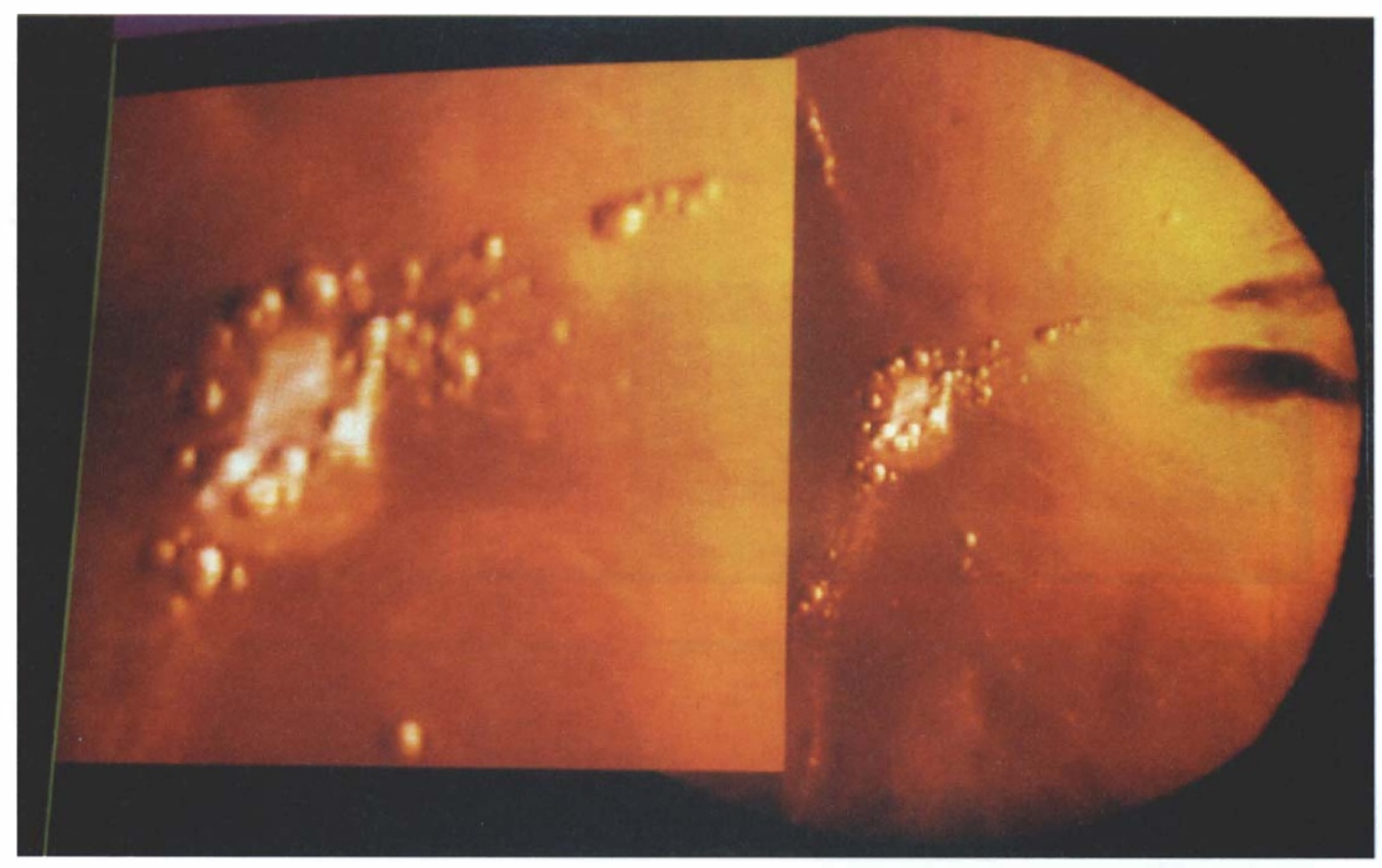

Fig. 5. Retro-illumination view of cortical vacuoles and spoke-like opacities (right-hand side) and a magnified area of the central vacuoles (left-hand side).

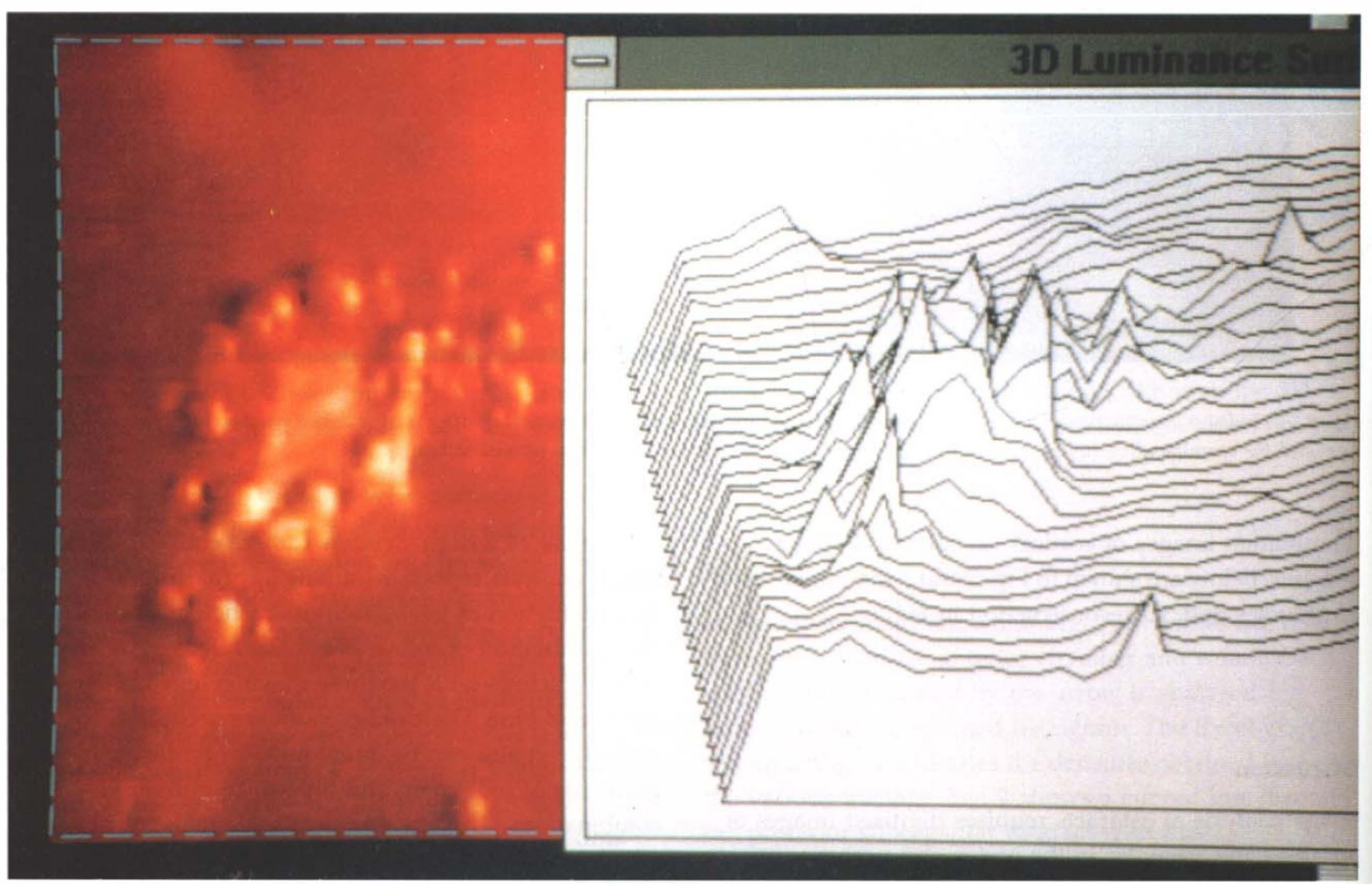

Fig. 6. Three-dimensional reconstruction of the cortical vacuoles in Fig. 5.

\section{References}

1. Sparrow JM, Bron AJ, Ayliffe W, Sparrow JM, Hill AR. The Oxford clinical cataract classification and grading system. Int Ophthalmol 1986;9:207-25.

2. Hockwin O, Dragomirescu V, Laser H. Measurement of lens transparency and of its disturbance by densitometric image analysis of Scheimpflug photographs. Graefes Arch Clin Exp Ophthalmol 1982;219:255-62.
3. Brown NAP, Bron AJ, Ayliffe W, Sparrow JM, Hill AR. The objective assessment of cataract. Eye 1987;1:234-46.

4. Cotlier E, Fagadau W, Cicchetti DV. Method for evaluation of medical therapy of senile and diabetic cataracts. Trans Ophthalmol Soc UK 1982;102:416-22.

5. Chylack LT, White O, Tung WH. Classification of human cataractous change by the American co-operative cataract research group method. Staged simplification of cataract classification. Invest Ophthalmol 1984;25:166-73. 
6. Chylack LT, Rosner B, Cheng HM, McCarthy D, Pennett M. Sources of variance in the objective documentation of human cataractous change with Topcon SL-45 and Neitz CTR retroillumination photography and computerised image analysis. Curr Eye Res 1987;6:1831-40.

7. Mayer H, Irion KM. New approach to area image analysis of Scheimpflug photos of the anterior eye segment. Ophthalmic Res 1985;17:106-10.
8. Niesel von P. Spaltlampenphotographie mit der Haag-Streit Spaltlampe 900. Ophthalmologica (Basel) 1966;151:489-504.

9. Maclean $\mathrm{H}$, Taylor $\mathrm{CI}$. An objective staging for cortical cataract in vivo aided by pattern analysing computer. Exp Eye Res 1981;33:597-602. 